\title{
High-intensity focused ultrasound (HIFU) ablation versus surgical interventions for the treatment of symptomatic uterine fibroids: a systematic review and meta-analysis
}

\author{
Lu Liu ${ }^{1}$, Baiying Lei $^{1}$, and Tianfu Wang ${ }^{1}$ \\ ${ }^{1}$ Affiliation not available
}

October 19, 2020

\begin{abstract}
Objective The purpose of this meta-analysis was to compare the effectiveness and safety of HIFU with surgical interventions for the treatment of symptomatic uterine fibroids in women according to the studies available in current literature. Main results A total of 10 studies involving 4450 women were included in our meta-analysis. Compared with surgery group, the reduction of uterine fibroid symptom (UFS) scores at 6- and 12-month follow-up were higher in HIFU group, with the overall MD -4.16 (95\% CI, -7.39 to $-0.94, \mathrm{P}=0.01$ ) and -2.44 (95\% CI, -3.67 to $-1.20, \mathrm{P}=0.0001$ ), respectively. The increase of quality-of-life (QoL) scores at 6- and 12-month follow-up were also higher in HIFU group, with the overall MD 2.13 (95\% CI, 0.86 to 3.14 , $\mathrm{P}=0.001)$ and 2.34 (95\% CI, 0.82 to $3.85, \mathrm{P}=0.003)$, respectively. Both of the duration of hospital stay and the time to return to work was significantly shorter in HIFU group, with the overall MD -3.41 (95\% CI, -5.11 to $-1.70, \mathrm{P}<0.0001)$ and -11.61 (95\% CI, -19.73 to $-3.50, \mathrm{P}=0.005$ ), respectively. The incidence of significant complications was significantly lower in HIFU group, with the overall RR 0.33 (95\% CI, 0.13 to $0.81, \mathrm{P}=0.02)$. The difference of incidence of adverse events, effective rate, symptom recurrence rate, re-intervention rate and pregnancy rate between HIFU and surgery were not statistically significant. Conclusion Compared with surgical interventions, HIFU ablation therapy leads to more significant alleviation of symptoms and improvement of QoL, quicker postoperative recovery and fewer significant complications.
\end{abstract}

\section{Hosted file}

HIFU20201017.pdf available at https://authorea.com/users/368380/articles/487524-highintensity-focused-ultrasound-hifu-ablation-versus-surgical-interventions-for-thetreatment-of-symptomatic-uterine-fibroids-a-systematic-review-and-meta-analysis

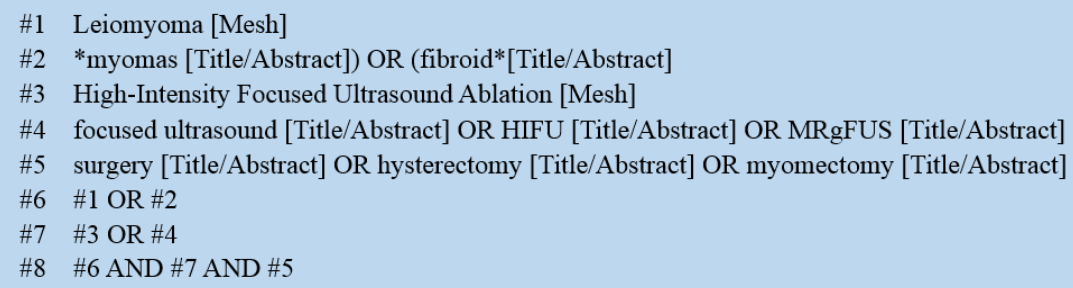




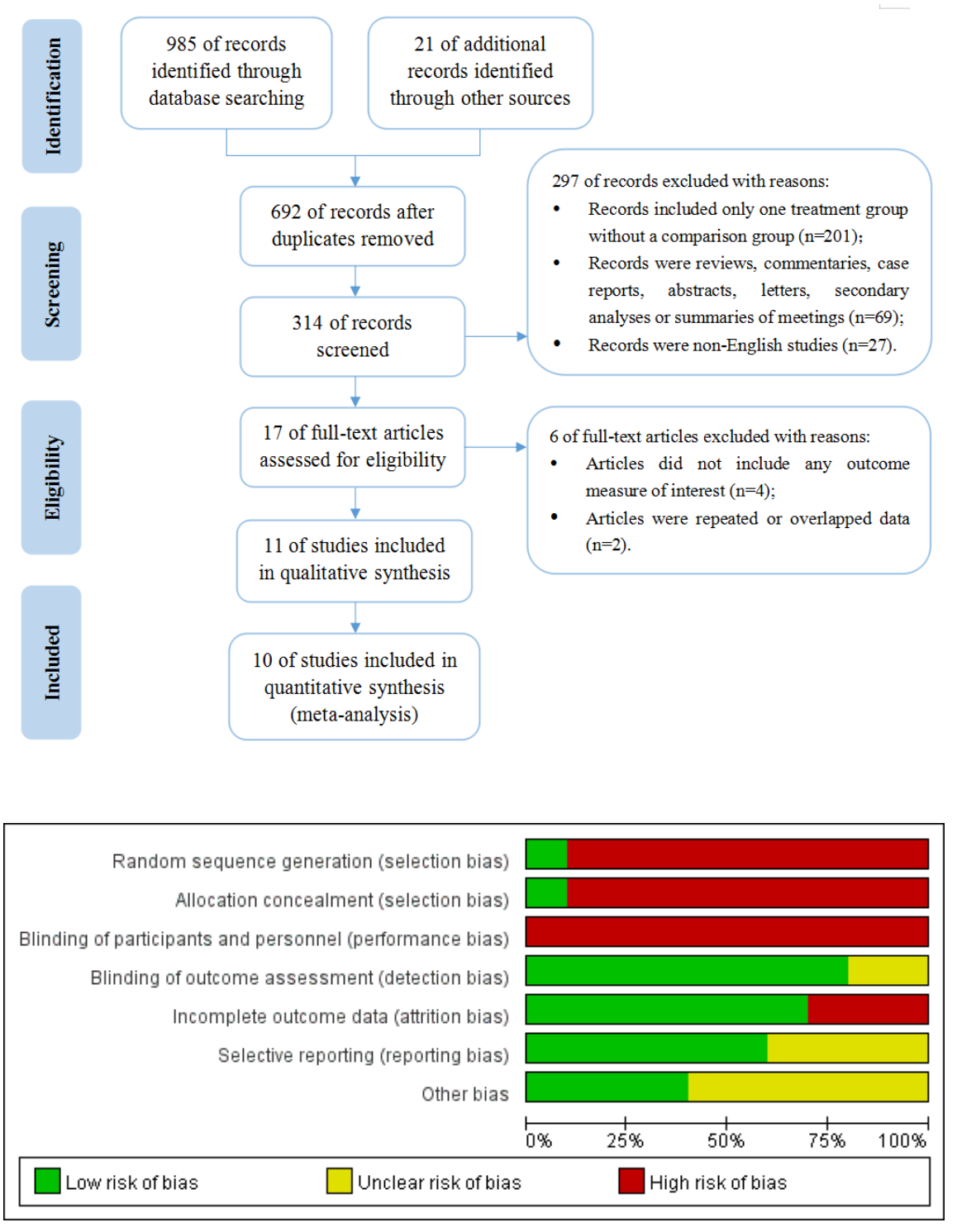

\begin{tabular}{|c|c|c|c|c|c|c|c|c|c|c|}
\hline $\begin{array}{l}\text { s } \\
\text { N } \\
\text { 品 }\end{array}$ & 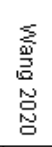 & 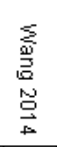 & $\begin{array}{l}5 \\
\stackrel{5}{\Xi} \\
\stackrel{3}{\Xi} \\
\stackrel{\Xi}{\Xi} \\
\omega\end{array}$ & 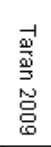 & 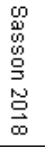 & 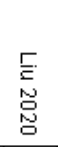 & 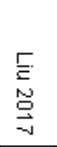 & $\begin{array}{l}\text { I } \\
\text { N } \\
\text { 怘 } \\
\text { Ong }\end{array}$ & 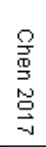 & \\
\hline (1) & (1) & (1) & + & (1) & (1) & (1) & (1) & (1) & (1) & Random sequence generation (selection bias) \\
\hline (1) & (1) & (1) & $\odot$ & (1) & (1) & (1) & (1) & (1) & (1) & Allocation concealment (selection bias) \\
\hline (1) & (1) & (1) & (1) & (1) & (1) & (1) & (1) & (1) & (1) & Blinding of participants and personnel (performance bias) \\
\hline 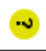 & $\odot$ & $\odot$ & $\odot$ & $\odot$ & $\odot$ & $\odot$ & $\sim$ & $\odot$ & + & Blinding of outcome assessment (detection bias) \\
\hline$\odot$ & $\odot$ & $\odot$ & (1) & $\odot$ & (1) & $\odot$ & $\odot$ & $\odot$ & (1) & Incomplete outcome data (attrition bias) \\
\hline$\odot$ & $\sim$ & $\odot$ & $\odot$ & $\sim$ & $\odot$ & $\odot$ & $\odot$ & $\sim$ & $\sim$ & Selective reporting (reporting bias) \\
\hline$\sim$ & $\odot$ & $\sim$ & 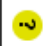 & + & $\sim$ & $\cdot$ & $\odot$ & $\sim$ & + & Other bias \\
\hline
\end{tabular}



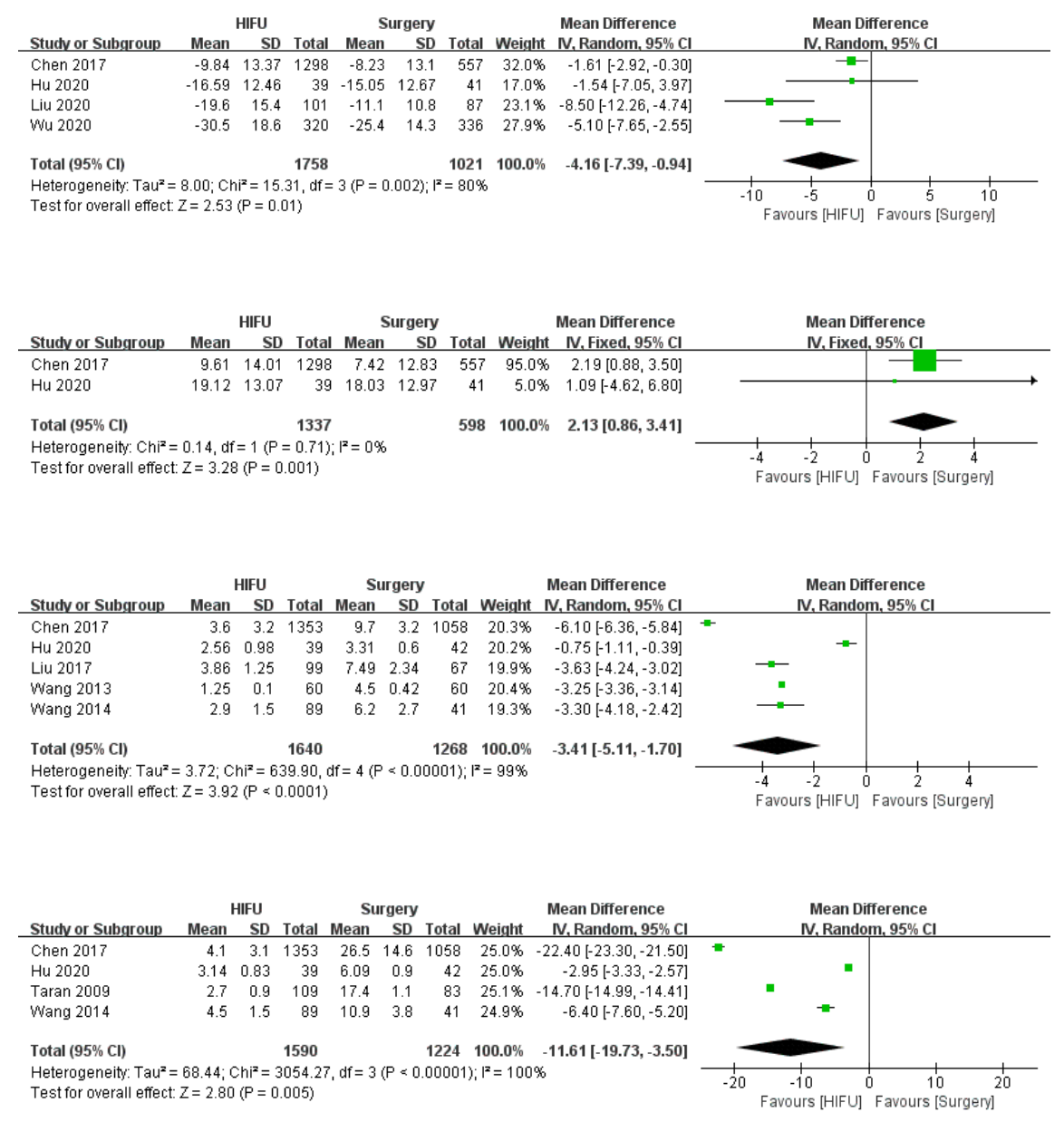

HIFU

Surgery Risk Ratio

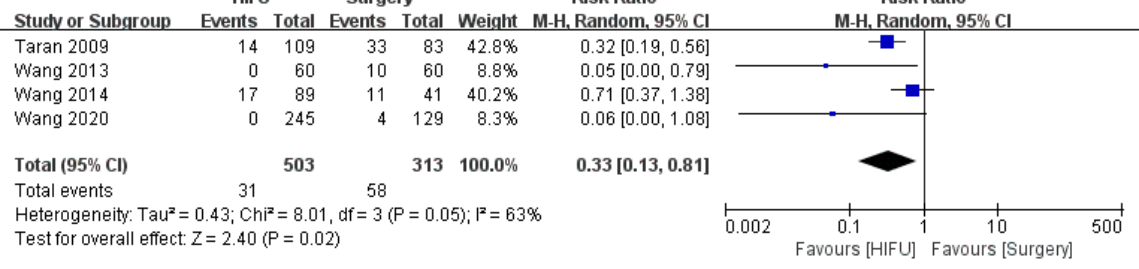

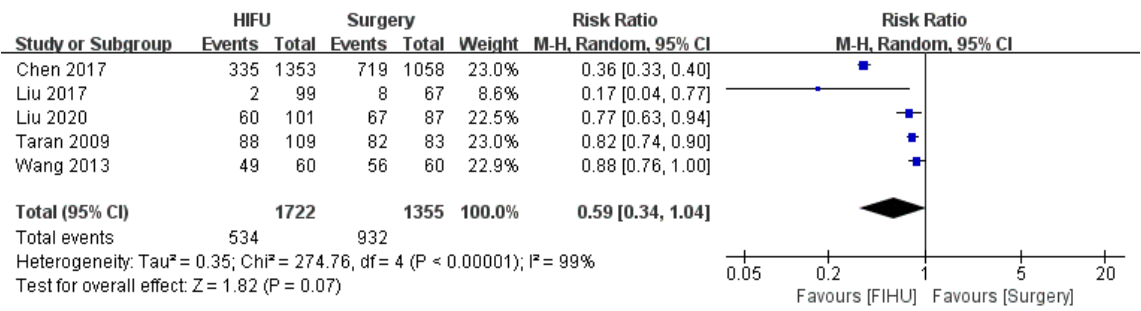




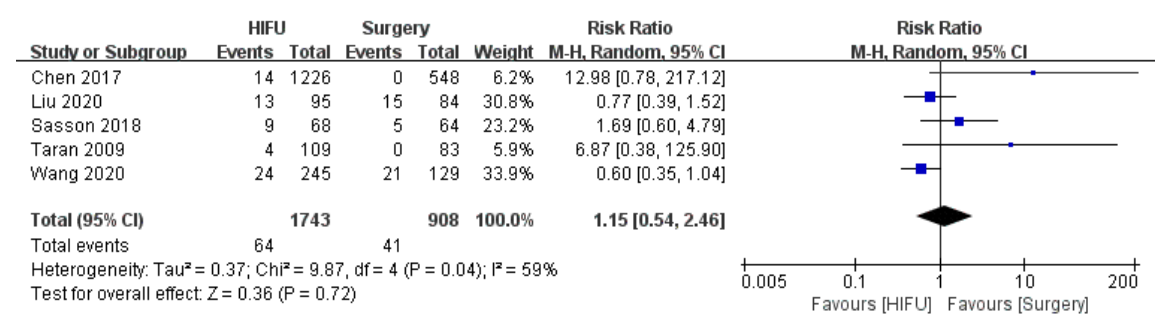

$\begin{array}{llll}\text { Experimental Control Risk Ratio } & \text { Risk Ratio }\end{array}$

Studv or Subgroup Events Total Events Total Weight M-H. Random, 95\% CI 3.1.1 HIFU vs Hysterectomy

Taran 2009

Subtotal $(95 \%$
Total events

$14 \quad 109$

Tonts Total Weight

Heterogeneity: Not applicable

Test for overall effect: $Z=3.98$ ( $P<0.0001)$

3.1.2 HIFU vs Uterus-sparing surgery

Wang 2013

Wang 2014

Wang 2020

Subtotal $(95 \% \mathrm{Cl})$

Total events

$\begin{array}{llll}109 & 33 \quad 83 & 428 \%\end{array}$

$0.32[0.19,0.56]$

M-H, Random, 95\% Cl

Heterogeneity: $\mathrm{Tau}^{2}=3.09 ; \mathrm{Chi}^{2}=7.37, \mathrm{df}=2(\mathrm{P}=0.03) ; \mathrm{I}^{2}=73 \%$

Test for overall effect: $Z=1.48(P=0.14)$

$\begin{array}{llll}\text { Total }(95 \% \mathrm{Cl}) & 503 & 313 & \mathbf{1 0 0 . 0 \%}\end{array}$

Total events 31

$\begin{array}{lllll}0 & 60 & 10 & 60 & 8.8 \%\end{array}$ $0.32[0.19,0.56]$

Heterogeneity: $\mathrm{Tau}^{2}=0.43 ; \mathrm{Chi}^{2}=8.01, \mathrm{df}=3(\mathrm{P}=0.05) ; \mathrm{I}^{2}=63 \%$

Test for overall effect: $Z=2.40(P=0.02)$

Test for subaroun differences: $\mathrm{Ch}^{2}=0.27 . \mathrm{df}=1(\mathrm{P}=0.60) . \mathrm{I}^{2}=0 \%$

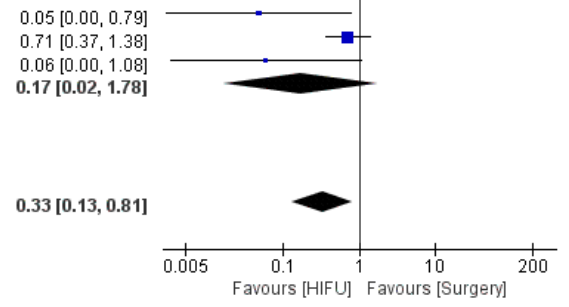

HIFU Surgery

Risk Ratio

Risk Ratio

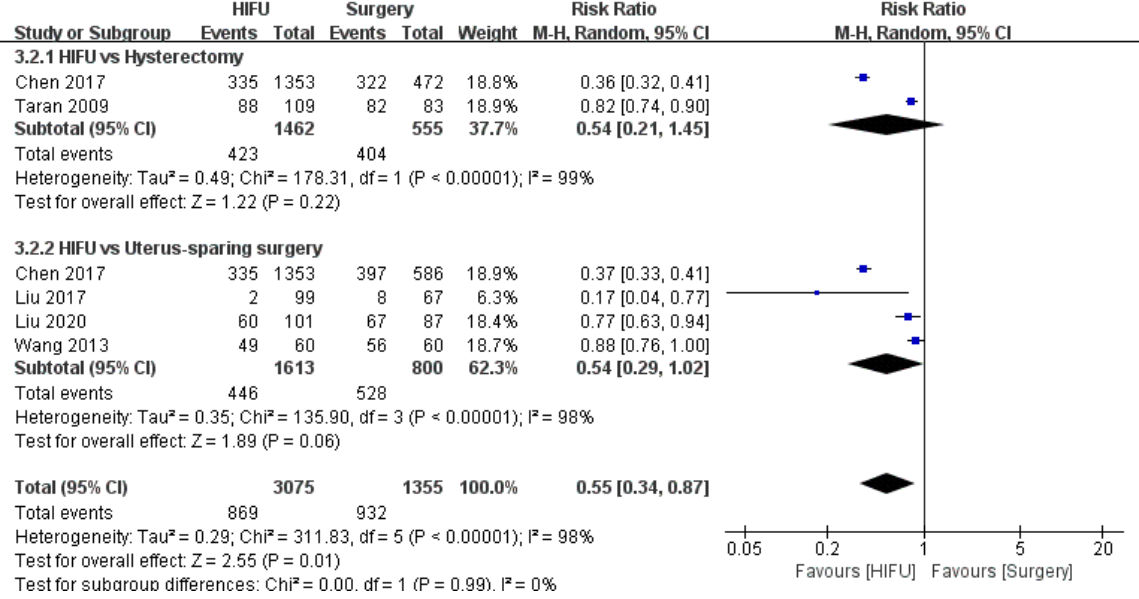




\begin{tabular}{|c|c|c|c|c|c|c|c|c|c|}
\hline \multirow{2}{*}{\multicolumn{2}{|c|}{$\begin{array}{l}\text { HIFI } \\
\text { Study or Subgroup } \\
\text { Events }\end{array}$}} & $\begin{array}{l}\text { IFU } \\
\text { s Total }\end{array}$ & \multicolumn{2}{|c|}{ Surgery } & \multicolumn{2}{|c|}{$\begin{array}{c}\text { Risk Ratio } \\
\text { Weight } \\
\text { M-H, Random, } 95 \% \mathrm{Cl}\end{array}$} & \multicolumn{3}{|c|}{$\begin{array}{c}\text { Risk Ratio } \\
\text { M-H, Random, } 95 \% \mathrm{Cl}\end{array}$} \\
\hline & & & & & & & & M-H, Kando & \\
\hline Chen 2017 & 3 & 1353 & 73 & 472 & $20.7 \%$ & $0.01[0.00,0.05]$ & & & 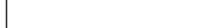 \\
\hline Taran 2009 & 9 & 109 & 8 & 83 & $21.2 \%$ & $0.86[0.35,2.13]$ & & 二 & \\
\hline Subtotal $(95 \% \mathrm{Cl})$ & & 1462 & & 555 & $42.0 \%$ & $0.11[0.00,8.48]$ & & & \\
\hline Total events & 12 & & 81 & & & & & & \\
\hline \multirow{2}{*}{\multicolumn{10}{|c|}{$\begin{array}{l}\text { Heterogeneity: } \mathrm{Tau}^{2}=9.45 ; \mathrm{Ch}^{2}=34.82, \mathrm{df}=1(\mathrm{P}<0.00001) ; \mathrm{I}^{2}=97 \% \\
\text { Test for overall effect: } Z=0.99(P=0.32)\end{array}$}} \\
\hline & & & & & & & & & \\
\hline \multicolumn{10}{|c|}{ 3.3.2 HIFU vs Uterus-sparing surgery } \\
\hline Chen 2017 & 3 & 1353 & 60 & 586 & $20.7 \%$ & $0.02[0.01,0.07]$ & & & \\
\hline Liu 2020 & 0 & 101 & 18 & 87 & $15.6 \%$ & $0.02[0.00,0.38]$ & & & \\
\hline Wang 2013 & 13 & 60 & 23 & 60 & $21.8 \%$ & $0.57[0.32,1.01]$ & & - & \\
\hline Subtotal $(95 \% \mathrm{Cl})$ & & 1514 & & 733 & $58.0 \%$ & $0.07[0.00,1.53]$ & & & \\
\hline Total events & 16 & & 101 & & & & & & \\
\hline \multirow{2}{*}{\multicolumn{10}{|c|}{$\begin{array}{l}\text { Heterogeneity: } \mathrm{Tau}^{2}=6.52 ; \mathrm{Chi}^{2}=37.21, \mathrm{df}=2(\mathrm{P}<0.00001) ;\left.\right|^{2}=95 \% \\
\text { Test for overall effect: } Z=1.68(\mathrm{P}=0.09)\end{array}$}} \\
\hline & & & & & & & & & \\
\hline Total $(95 \% \mathrm{Cl})$ & & 2976 & & 1288 & $100.0 \%$ & $0.09[0.01,0.67]$ & & & \\
\hline Total events & 28 & & 182 & & & & & & \\
\hline \multirow{2}{*}{\multicolumn{7}{|c|}{$\begin{array}{l}\text { Heterogeneity: } \mathrm{Tau}^{2}=4.75 ; \mathrm{Chi}^{2}=74.33, \mathrm{df}=4(\mathrm{P}<0.00001) ; \mathrm{I}^{2}=95 \% \\
\text { Test for overall effect: } Z=2.35(\mathrm{P}=0.02) \\
\text { Test for subaroun differences: } \mathrm{Chi}^{2}=0.03 . \mathrm{df}=1(\mathrm{P}=0.87) . \mathrm{I}^{2}=0 \%\end{array}$}} & 0.002 & 0.1 & 500 \\
\hline & & & & & & & & & \\
\hline
\end{tabular}

